\title{
Weite Wege zum Kind
}

\author{
Reproduktionstourismus im Ausland Etwa jedes siebte Paar in Deutschland \\ bleibt ungewollt kinderlos. Mehr als 100.000 Paare pro Jahr unterziehen sich \\ deshalb einer Kinderwunschbehandlung. Aber nicht einmal ein Drittel der Be- \\ handlungen führt zum erhofften Erfolg. Wenn alle medizinischen Optionen in \\ Deutschland auf dem Weg zum eigenen Kind ausgeschöpft sind, empfehlen \\ Ärzt*innen den Weg ins Ausland.
}

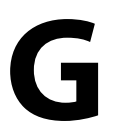
esetzlich ist im Ausland reproduktionsmedizinisch mehr erlaubt als hier bei uns. Ein durchaus legitimer Weg, der allerdings Verwandten, Freunden und Kollegen immer noch gerne verschwiegen wird. Warum ist das so? Welche Hürden müssen überwunden werden und welche Chancen bietet der Reproduktionstourismus ins Ausland?

\section{Recht und Gesetz}

In Deutschland regeln vor allem das Embryonenschutzgesetz (ESchG) und das Gesetz zur Präimplantationsdiagnostik die An-

\section{- Im internationalen Vergleich gehört Deutschland zu den Ländern mit den strengsten Richtlinien im Rahmen der Reproduktionsmedizin.}

wendung von Reproduktionstechniken sowie den Umgang mit Embryonen. Nach dem Gesetz erlaubte Methoden zur künstlichen Herbeiführung einer Schwangerschaft sind die Übertragung von Samen des Partners (homologe Insemination), die In-vitro-Fertilisation (IVF) und die Intrazytoplasmatische Spermieninjektion (ICSI) durch speziell ausgebildete Ärztinnen und Ärzte, die Übertragung von bis zu drei befruchteten Eizellen oder Embryonen in einem Zyklus, das Einfrieren (Kryokonservierung) von Eizellen im Vorkernstadium, die Übertragung von Samen eines Spenders (heterologe oder donogene Insemination) und die Präimplantationsdiagnostik nach ausführlicher medizinischer und psychosozialer Beratung. Hingegen gesetzlich verbotene Methoden sind die Eizellspende, die Leihmutterschaft, Experimente an Embryonen, die Geschlechterauswahl sowie die Verwendung von Samen bereits Verstorbener.

Deutschland gehört damit im internationalen Vergleich zu den Ländern mit den strengsten Richtlinien im Rahmen der Reproduktionsmedizin. Längst Zeit, etwas zu ändern, finden nicht nur
Patient*innen, sondern auch Reproduktionsmediziner*innen und Politiker*innen. Bereits seit einigen Jahren wird eine Weiterentwicklung des ESchG diskutiert, eine Gesetzesänderung ist bislang aber noch ausgeblieben. Der Fortschritt in der Reproduktionsmedizin schreitet schnell voran. Das Risiko für Mehrlingsschwangerschaften konnte deutlich gesenkt werden. Zudem steigt die Zahl der gleichgeschlechtlichen Paare, die eine Familie gründen möchten und dazu medizinische Hilfe benötigen. Nicht zuletzt lassen sich Paare mit ihrem Kinderwunsch immer mehr Zeit, was das Risiko von Fertilitätsstörungen mit steigendem Lebensalter automatisch erhöht und dazu führt, dass sie immer häufiger moderne Kinderwunschbehandlungen in Anspruch nehmen.

\section{Adoption, Pflegeelternschaft oder ins Ausland?}

Wenn die medizinischen Wege hierzulande ausgeschöpft sind, bleibt Paaren nur die Möglichkeit einer Adoption oder Pflegeelternschaft. Der Wunsch nach dem eigenen Kind bleibt aber meist ungebrochen und führt viele in andere Länder. Es macht sich niemand strafbar, der sich im Ausland mit in Deutschland verbotenen Methoden wie einer Eizellspende behandeln lässt. Es gelten die Regelungen des jeweiligen Landes. Auch Frauenärzt*innen, die die Behandlung in Deutschland weiter betreuen, können nicht strafrechtlich belangt werden. Schätzungsweise rund 15.000 Paare reisen jährlich zur Kinderwunschbehandlung ins Ausland, wo alternative reproduktionsmedizinische Behandlungen möglich sind. Anders als in Deutschland ist in den meisten europäischen Staaten beispielsweise das Verfahren des elektiven Single Embryo Transfers (eSET) Standard. Dabei werden die in-vitro gezeugten Embryonen nach bestimmten morphologischen Kriterien ausgewählt, die eine Implantationsfähigkeit und einen Behandlungserfolg erwarten lassen.

In Österreich etwa ist die Samenspende auch für lesbische Paare erlaubt. Zudem darf die In-vitro-Fertilisation (IVF) mit dem Samen Dritter durchgeführt werden. Eine Eizellspende ist dort ebenso legal wie eine Untersuchung des Embryos vor der Einpflanzung (PID). Auch in Tschechien und Spanien dürfen Eizellspenden von anonymen Spenderinnen vermittelt und eingepflanzt werden. Je 


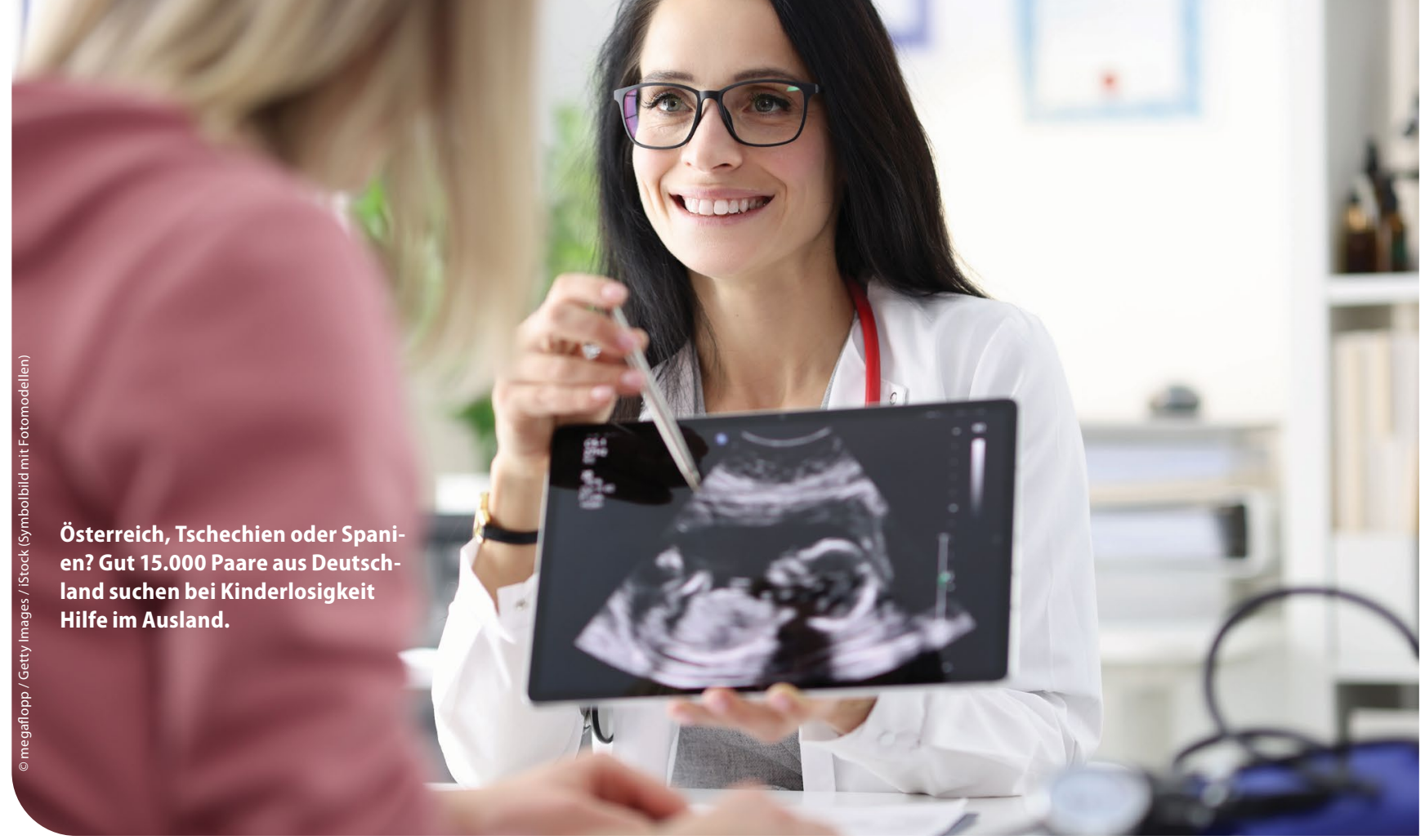

nach Land liegen die Kosten für die künstliche Befruchtung bei 3.000 bis 15.000 Euro.

In Tschechien gibt es 42 Reproduktionskliniken, in denen Schätzungen zufolge rund 30.000 Paare, vor allem aus dem westeuropäischen Ausland, jedes Jahr Hilfe suchen. In Tschechien ist es günstiger und die medizinischen Möglichkeiten sind besser. Eine Erfolgsquote von durchschnittlich $70 \%$ macht vielen Hoffnung. Hier werden beispielsweise Eizellen im Labor untersucht und lebensun-

\section{- Jedes Paar muss für sich entscheiden, inwie- weit es eine Behandlung im Ausland körper- lich, finanziell, organisatorisch und vor allem auch emotional verkraftet.}

fähige oder genetisch defekte Eizellen direkt aussortiert, bevor sie der Frau eingesetzt werden. Das ist in Deutschland nicht erlaubt.

Eine nichtkommerzielle Leihmutterschaft ist unter anderem in Frankreich, Großbritannien, Spanien und den Niederlanden erlaubt. In der Ukraine und den USA ist auch eine kommerzielle Leihmutterschaft legal. Wenn man im Ausland eine Leihmutterschaft nutzt, wird es in Deutschland aber geduldet. Die (genetische) Mutter muss im Nachhinein ihr Kind in Deutschland noch einmal selbst adoptieren, ein oft langwieriger und nervenaufreibender Prozess. In Deutschland gilt die Würde jedes Menschen als unantastbar und man befürchtet, dass mit einer Leihmutterschaft oder gegebenenfalls mit einer Eizellspende, das Kind zu einer reinen Ware verkommen könnte. Diesem Eindruck kann man sich nicht ganz verwehren und führt bei deutschen Paaren auch nicht selten zu ethisch-moralischen Konflikten.

\section{Nachgefragt - Die Geschichte von einer Betroffenen}

Viele Paare, die sich dazu entscheiden, ins Ausland zu gehen, haben oft einen langen Leidensweg hinter sich. Zahlreiche gescheiterte Versuche mit kräftezehrenden Behandlungen, emotionalen Achterbahnfahrten und dem Gefühl, dass weitere Chancen, in Deutsch- land schwanger zu werden, aussichtslos scheinen. Nur wenige sind bereit, darüber offen zu reden. Aber warum?

Sina (Name ist der Redaktion bekannt), 36 Jahre, aus Niedersachsen, hat sich bereit erklärt, ihre Geschichte zu erzählen. Sie sagt, sie hätten aus ihrem Weg zwar kein Geheimnis gemacht, aber sie hätten ihn auch nicht jedem erzählt. Auch einigen Angehörigen aus dem engsten Familienkreis nicht: „Wer erzählt schon, wann und wo das Kind entstanden ist". Dennoch sei es wichtig, das Thema Kinderwunschbehandlung im Ausland mehr in die Öffentlichkeit zu tragen, denn es seien so viele davon betroffen. Weil man sich in einer gefühlten Grauzone bewege, überwiege aber noch immer die Scham, anderen davon zu berichten, meint sie.

2017 erlitt sie eine Fehlgeburt und danach „hat es einfach nicht mehr geklappt", sagt sie. Das Paar entschied sich für eine Kinderwunschbehandlung. Es folgten mehrere Inseminationen ab Januar 2020, leider alle ohne Erfolg. Immer an ihrer Seite, ihr Gynäkologe, der dafür bekannt ist, Frauen auf ihrem Weg zum Wunschkind vertrauensvoll zu begleiten. Er war es auch, der Sina und ihrem Mann vorschlug, eine Kinderwunschklinik in Prag aufzusuchen. Aufgrund der Anamnese seien sie prädestiniert für eine Behandlung dort; 98\% der Frauen, die er dort hinschicken würde, würden schwanger und 95\% würden es auch bleiben. Damit war die Entscheidung gefallen. Das Rezept für die Medikamente aus Prag erhielt sie direkt von ihrem Gynäkologen. Diese nahm sie für ihren Zyklus im Juni ein. Ende Juni ging es dann nach Tschechien zur Punktion in die Klinik in Prag. Mit fünf weiteren Frauen aus unterschiedlichen Ländern im Zimmer, wartete sie auf ihren Eingriff. Immer in der Nähe, eine Dolmetscherin.

Es konnten bei ihr 29 Bläschen punktiert werden, von denen im Labor 14 Eizellen erfolgreich mit dem Sperma ihres Mannes befruchtet wurden und heranreifen konnten. Fünf Tage später erfolgte der Transfer von zwei Blastozysten. Dafür musste sie nach dem Eingriff noch eine Stunde auf dem Bauch liegen und kam wieder ins Gespräch mit einer Bettnachbarin aus Deutschland. Die übrigen Eizellen ließen sie einfrieren, für einen möglichen weiteren Versuch oder ein weiteres Kind. Eine der beiden Blastozysten reifte heran. Sina war schwanger. 
Während ihrer gesamten Schwangerschaft wurde sie von ihrem Gynäkologen engmaschig betreut. Nicht nur rein medizinisch, auch emotional. Sie sagt, sie habe sich bei ihm rundherum gut aufgehoben gefühlt. Für sie sei es wichtig gewesen, dass er auch außerhalb der Sprechstunden via E-Mail für sie erreichbar war, wenn sie ein komisches Gefühl im Bauch hatte und sich Sorgen um ihre Schwangerschaft machte. Erst ab der 16. Schwangerschaftswoche bemühte sie sich um eine Hebamme, denn zu groß war die Angst, dass wieder etwas schief gehen würde. Jasmin, ihre Hebamme, war ihr Jackpot, wie Sina heute sagt. Regelmäßige Treffen zur Fußmassage mit ausgiebigen Gesprächen schafften Vertrauen und gaben ihr Sicherheit. Mitte März 2021 war es dann soweit. Sina brachte ihre kleine Tochter in einem Krankenhaus in der Nähe ihres Wohnortes zur Welt. Die Geburt verlief schnell - etwas zu schnell - aber nach kurzen Anlaufschwierigkeiten konnten Mutter und Tochter nach wenigen Tagen fit nach Hause entlassen werden.

\section{- Paare müssen darauf gefasst sein, die Kosten ihrer reproduktionsmedizinischen Behandlung selber tragen zu müssen.}

Im Wochenbett war ihre Hebamme immer an ihrer Seite und nahm der jungen Mutter viele Ängste und den Druck, alles richtig machen zu müssen. Ein Happy End. Das hat Sina auch der Reproduktionsklinik in Prag zurückgemeldet. Diese ist natürlich daran interessiert, zu erfahren, ob ihre Behandlungen zum Erfolg geführt haben. Ob sie es wieder tun würde? Auf jeden Fall, denn das Ergebnis zähle, sagt sie. Ihre eingefrorenen Eizellen kann sie jederzeit einsetzen lassen. Ein Anruf genügt. Noch nicht jetzt, aber vielleicht schon bald.

\section{Und die Kostenfrage? Kasse oder privat?}

Die Kosten für Sinas Behandlung beliefen sich auf rund 5.000 Euro. Ein finanzieller Aufwand, den man kalkulieren kann, denn die jeweiligen Preise sind auf den meisten Internetseiten vieler Kinderwunschkliniken transparent aufgeführt. Ob diese Kosten allerdings von den Krankenkassen in Deutschland übernommen werden, ist nicht immer sicher. Grundsätzlich werden Kinderwunschbehandlungen auch im Ausland von den Krankenkassen bezahlt, wenn sie nach deutschem Recht erlaubt sind. Es entscheidet immer der Einzelfall und Paare müssen darauf gefasst sein, die Kosten ihrer reproduktionsmedizinischen Behandlung selber tragen zu müssen. Zumal viele im Ausland gewählte Methoden - beispielsweise eine Leihmutterschaft oder eine Eizellspende - in Deutschland nicht erlaubt sind und demzufolge nicht bezahlt werden.

Die Krankenkassen tragen normalerweise die vollen Kosten für die Diagnostik, um die Ursachen für die ungewollte Kinderlosigkeit festzustellen. Für eine künstliche Befruchtung übernehmen die gesetzlichen Krankenkassen in Deutschland bei verheirateten Paaren mit ungewollter Kinderlosigkeit 50\% der Kosten für insgesamt acht Zyklen einer Insemination ohne vorherige hormonelle Stimulation, drei Zyklen einer Insemination mit hormoneller Stimulation und drei Zyklen einer IVF-Behandlung oder drei Zyklen einer ICSI-Behandlung. Hinzu kommen Zuschüsse des Bundes und der
Länder. Die Kostenübernahme ist an bestimmte Voraussetzungen geknüpft: Neben der Einhaltung des rechtlichen Rahmens muss der behandelnde Arzt der Überzeugung sein, dass „hinreichend Aussicht auf Erfolg“ mit der Therapie besteht. Die Paare müssen verheiratet sein. Auch das Alter spielt eine Rolle: die Partner müssen mindestens 25 Jahre alt sein. Die Frau darf nicht älter als 39 Jahre, der Mann nicht älter als 49 Jahre sein. Außerdem müssen sich die Partner medizinisch und psychosozial beraten lassen und einen HIV-Test machen. Bei den privaten Krankenversicherungen entscheidet der individuelle Vertrag über den Umfang der Kostenübernahme.

\section{Selbstverständlich will ich ein Kind}

Kinder sind für viele Menschen selbstverständlich. Viele andere begleitet das Thema einer ungewollten Kinderlosigkeit jeden Tag. Alles wird diesem Ziel untergeordnet. Wenn der Wunsch so groß ist und die Chancen im Ausland auf ein eigenes Kind besser oder überhaupt erst möglich sind, liegt der Schritt, ins Ausland zu gehen, auf der Hand. Und dennoch muss jedes Paar für sich entscheiden, inwieweit es eine Behandlung im Ausland körperlich, finanziell, organisatorisch und vor allem auch emotional verkraften kann und will.

\section{(1) FAZIT}

Für die betroffenen Paare spielt neben dem Behandlungsverfahren, der Auswahl des Landes, der Klinik, der möglichen Überwindung von Sprachbarrieren, juristischen Regelungen im jeweiligen Behandlungsland und in Deutschland auch eine Rolle, wen sie aus ihrem Umfeld in die Kinderwunschbehandlung einweihen.

Viele Paare, Reproduktionsmediziner*innen und Hebammen wünschen sich mehr Freiheiten in der Kinderwunschbehandlung in Deutschland. Das Gefühl, etwas nicht Gesellschaftskonformes, vielleicht sogar Unerlaubtes zu tun, könnte unter anderem mit einer Reform des Embryonenschutzgesetzes überwunden werden.

Neben den betreuenden Ärzt*innen kommt den Hebammen eine besondere Bedeutung zu. Den Schwangeren ist es wichtig, engmaschig begleitet zu werden, um nach vielen Enttäuschungen behutsam Vertrauen in ihren Körper und die Schwangerschaft aufzubauen. Aber auch nach der Geburt geht es darum, ihnen im Umgang mit ihrem Kind, Ängste zu nehmen.

Schlüsselwörter: Kinderwunsch, Reproduktion, Kinderwunschklinik, Schwangerschaft im Ausland 